\title{
Temporalité queer. Résistance et désir
}

\author{
Olivier Ducharme
}

Les articles portant sur le queer se font rares dans le monde francophone, et bien souvent, lorsqu'il en est question, ce n'est qu'en passant ou dans le contexte d'études féministes. Malgré la traduction, ces dernières années, de plusieurs textes importants, le développement des études queer francophones, en tant que champ d'études indépendant, demeure marginal et souffre d'un manque de visibilité. L'une des conséquences de cette situation est une simplification et une réduction des problématiques de ce champ d'études : la théorie queer se pose comme une théorie du genre et de la sexualité. Perçue de cette manière, la théorie queer devient un «avatar» (Berger 13) de la théorie du genre et une branche des études portant sur la sexualité.

Depuis plusieurs années, le champ des études queer s'est cependant élargi pour faire place à une série de réflexions sur des sujets aussi diversifiés que l'histoire (Dinshaw 1999, Nealon 2001, Cvetkovich 2003, Love 2007), le racisme (Ferguson 2004, Puar 2007, Eng 2010, Reddy 2011), le néolibéralisme (Duggan 2003, Puar 2007, Eng 2010), la vie des affects (Sedgwick 2003, Cvetkovich 2003, Love 2007, Ahmed 2010), etc. Au lieu de percevoir d'un mauvais œil cette croissance thématique ${ }^{1}$, nous croyons que ces réflexions permettent de mettre au jour les potentialités propres à ce champ d'études. Parmi les thématiques abordées ces dernières années, il en est une qui nous semble particulièrement importante et digne d'être au centre de nos réflexions : la question de la temporalité. Nous assistons depuis peu à un véritable «tournant temporel dans les études queer» (Dinshaw, How Soon 34), plusieurs articles et ouvrages (Halberstam 2005, Ahmed 2006, Freccero 2006, Muñoz 2009, Freeman 2010, McCallum et Tuhkanen 2011, Edelman 2013) faisant de la temporalité leur première source de réflexion. Si cette thématique retient l'attention de maintes théoricien-ne-s queer, la raison en est bien simple : la temporalité nous fait accéder à l'épicentre de la queerité. Loin de symboliser un détail ou une note, la temporalité donne plutôt la clé pour saisir la signification de l'un des aspects centraux des études queer : la

\footnotetext{
${ }^{1}$ Nous pensons ici à la question posée par Janet Halley et Andrey Parker : «Est-ce que des travaux ne portant pas explicitement au sujet de la sexualité peuvent être qualifiés de queer? » (Halley et Parker 2)
} 
queeritude est un concept dynamique, toujours porté par un mouvement qui le mène à ne pas rester en place et à créer de nouvelles possibilités. Ce mouvement vers l'avant signifie une ouverture vers un futur indéterminé et empreint de potentialités. Comme nous le verrons, le dynamisme propre à la queeritude se dévoile dans un acte de résistance par rapport à l'hétéronormativité et à une temporalité dont la principale caractéristique est de privilégier la répétition et la reproduction.

Le récent «tournant temporel» des études queer a permis de mettre au jour trois éléments importants que nous tenterons d'expliciter dans cet article: tout d'abord, que le temps dans lequel nous vivons actuellement, loin d'être une notion anhistorique ou éternelle, prend racine dans l'hétéronormativité. Ensuite, que la temporalité hétéronormative doit être envisagée comme ce qui prend vit au cœur même de l'existence corporelle de chaque subjectivité. Finalement, que la temporalité queer s'incarne dans un désir et une pratique apparaissant dans un acte de résistance. La visée de cet article est de démontrer que la temporalité queer se comprend fondamentalement en tant que résistance, et qu'au cœur de celle-ci se déploie la création de nouvelles possibilités temporelles.

\section{Négativité et résistance}

Avant même d'amorcer notre analyse, nous aimerions souligner un point important que nous devrons garder en tête tout au long de l'article : nous analysons le terme «queer» en tant que concept philosophique et non pas en tant que synonyme des différentes communautés LGBTQ ou des pratiques sexuelles «marginales ». Nous croyons qu'une réflexion au sujet de la signification du terme «queer » est plus que souhaitable pour éviter les malentendus et permettre à ce terme, d'origine anglophone, de traverser les frontières linguistiques pour devenir un concept politique de première importance ${ }^{2}$. Ce faisant, nous ne cherchons pas à minimiser l'accès privilégié de ce terme à la communauté LGBTQ ou au régime des identités et pratiques sexuelles marginales; nous nous efforçons plutôt d'interroger la valeur conceptuelle et les potentialités de la queeritude. Il faut toujours garder en tête que si ce concept a aujourd'hui une importance et qu'il peut servir à identifier certaines personnes et communautés, c'est parce qu'il a été employé en tout premier lieu dans une lutte politique pour contrer la propagation du virus VIH/SIDA ( $c f$. Gould). « Ce qui est souvent oublié », écrit Michael Warner, «est que le terme [queer] provient de la politique locale-populaire [grass-roots politics] avant d'être une théorie. » (Warner, "Queer and Then », non paginé) Nous ne devons jamais oublier le contexte historique dans lequel émerge la signification contemporaine

\footnotetext{
${ }^{2}$ Pour une discussion de l'impossibilité de traduire le terme « queer », $c f$. Bourcier.
} 
de «queer» et sa transformation conceptuelle au fil des ans ${ }^{3}$. Mais il est également possible de s'approprier ce terme et d'en faire un concept philosophique à part entière. C'est ce que nous nous proposons de faire en nous penchant sur l'un des aspects qui nous semble essentiel à sa compréhension, c'est-à-dire la temporalité. Avant d'aborder de front cette question, nous allons d'abord nous tourner vers le concept de résistance, qui servira de fondement à notre analyse, et son lien avec le concept de « queer ».

Paradoxalement, deux des ouvrages les plus influents qui ont permis, en 1990, de développer un champ de réflexion spécifique pour les études queer, Trouble dans le genre de Judith Butler et Épistémologie du placard d'Eve Kosofsky Sedgwick, ne se servent pas du terme « queer», ou du moins n'en font pas mention en tant que concept central à leurs travaux ${ }^{4}$. Il faut attendre la publication, en 1993, de l'article «Queer and Now » de Sedgwick et de l'ouvrage Bodies that Matter (Ces corps qui comptent) de Butler, pour enfin trouver une réflexion au sujet du terme «queer». Se fondant sur une critique de l'identité du genre et de l'orientation sexuelle, thématiques qui sont au fondement de leurs ouvrages précédents, Butler et Sedgwick soulignent le fait qu'au cœur de cette identité et de cette orientation se dévoile une négativité, c'est-à-dire un élément que nous ne pouvons pas saisir ou conceptualiser. La négativité qualifie l'action de la queeritude en tant que dissonance, défaillance et excès d'une «signification monolithique. » (Sedgwick, «Queer and Now » 7) La queeritude échappe à notre saisie et demeure ainsi en marge de notre compréhension. La négativité du queer fait en sorte que l'identité du genre ou de l'orientation sexuelle ne se referme jamais sur soi-même pour devenir homogène et sans surprise. Butler insiste, quant à elle, sur la multiplicité des significations que nous pouvons accorder à l'action de la queeritude : "Si le terme "queer" doit être un site de contestation collective, le point de départ de réflexions historiques et d'imaginations futures, il devra rester ce qui, dans le présent, n'est jamais entièrement possédé par personne, mais ne peut qu'être réutilisé, tordu, rendu étrange [queered] par rapport à un usage antérieur, et en vue de fins politiques urgentes et proliférantes. » (Butler, Corps 30) L'altération continuelle du sens du terme «queer » rend problématique l'exercice de vouloir définir une fois pour toutes ce concept et de lui donner une

\footnotetext{
${ }^{3}$ Il ne faut pas oublier ce que Lisa Duggan écrivait, en 1991, au sujet des études queer naissantes: "Le défi pour la théorie queer telle qu'elle émerge présentement de son ghetto académique est de s'engager intellectuellement dans un projet politique en employant dans le meilleur sens la "théorie", tout en évitant le jargon et l'obscurantisme dans le plus mauvais sens de 1'“académie". » (Duggan 161)

${ }^{4}$ Pour être plus précis, Butler emploie le terme queer à une seule occasion (Trouble 240) et Sedgwick, à quatre (Épistémologie 185, 212).
} 
signification positive, lui qui aurait toutes les caractéristiques pour rester à jamais une notion rebelle.

Ainsi, la difficulté principale qui se dresse devant nous lorsque nous tentons de définir la queerité du «queer» est qu'il se déploie à chaque fois en tant que négativité. Encore plus rebelle que tout rebelle, le queer devient effectif seulement lorsqu'on le situe comme négation de ce qui existe. Seule la négativité nous permet donc d'approcher la «nature » du queer, totalement indéterminée et insaisissable.

Le théoricien queer qui a le plus insisté sur la négativité est sans contredit Lee Edelman qui, dans son article «Le futur est un truc de gosse », a le plus poussé les conséquences d'une négativité queer : "de même le queer doit insister à jeter le trouble, à produire l'étrangeté de l'organisation sociale comme telle — jeter le trouble, donc, et produire l'étrangeté de nous-mêmes et de notre investissement dans une telle organisation. Car la queerité ne peut jamais définir une identité; elle ne peut jamais que l'inquiéter. »(Edelman 306) Deux éléments essentiels sont offerts dans ce passage : tout d'abord, la négativité du queer devient réelle uniquement en raison de sa position dans un système de normes particulier; et c'est en tenant compte de cette position obligatoire du queer que nous pouvons affirmer, dans un second temps, que «le queer ne peut jamais se définir comme identité ». Ce dernier point est excessivement important : la négativité radicale du queer fait de lui un être qui est rebelle à lui-même, refusant de se donner une identité qui lui est propre.

Cette posture anti-identitaire, qui a fait la renommée des études queer depuis ses tout débuts, n'a jamais été aussi clairement énoncée et radicalisée que sous la plume d'Edelman. Il faut immédiatement noter, cependant, que ce n'est pas la totalité du champ des études queer qui accepte la posture radicalement négative proposée par Edelman. Nous devons plutôt admettre que celui-ci fait figure de marginal en proposant une telle négativité et en refusant toute position positive. Sans retomber dans une recherche purement positive du terme « queer », c'est-à-dire sans chercher à donner une définition monolithique, la majorité des théoriciennes queer tendent à admettre que la négativité du queer mène à une positivité.

David M. Halperin résume bien la position problématique (négative et positive) dans laquelle se trouve le «queer», lorsqu'il écrit dans son ouvrage Saint Foucault que le queer se comprend « de manière oppositionnelle et relationnelle et non nécessairement en terme de réalité stable. Non pas comme une positivité mais comme un positionnement. Non pas comme un état [not as a thing] mais comme une résistance à la norme. » (Halperin, Saint Foucault 80) D'après lui, une personne doit se positionner de manière négative par rapport à une norme pour être qualifiée de «queer». Pour reprendre les ses mots, le queer n'est pas 
un «état » [thing], il ne représente pas une identité, car il se comprend à chaque instant uniquement en raison de sa position négative dans un système de normes. Défini en tant que négativité, la queerité ressemble davantage à un élément perturbateur dont le seul aspect positif se dévoile dans le fait qu'il vient mettre le trouble.

Halperin ne s'en tient pas uniquement à une définition négative de la queerité, il insiste pour y retrouver un aspect positif à travers la forme de la résistance : «la résistance à la normativité n'est pas purement réactive, négative ou destructrice; elle est aussi positive, dynamique et créatrice. » (80) L'opposition négative propre à la dynamique queer porte ainsi à la création de nouvelles manières de s'identifier soi-même et de nouvelles manières de vivre. La résistance queer déploie une double stratégie : elle s'oppose (négation) à un système de normes tout en créant (positif) de nouvelles manières de vivre.

L'élaboration d'une résistance queer ne s'applique pas exclusivement à l'analyse de Halperin; au contraire, elle est également présente chez la majorité des théoricien-nes queer (Muñoz, Halberstam, Duggan, Butler, Ahmed, etc.). Même si la plupart de ces auteur-e-s n'emploient pas explicitement le terme de « résistance » pour décrire l'un des aspects essentiels au travail de la queeritude, tous se fondent sur la logique d'une négativité positive, c'est-à-dire une négativité qui se déploie en tant que création.

$\mathrm{La}$ résistance queer recèle cependant encore une autre caractéristique essentielle. Pour saisir et comprendre toute la portée de la résistance queer, nous devons nous tourner vers la description de la résistance, proposée par Michel Foucault. Tout lecteur de Foucault aura remarqué que l'analyse que Halperin donne de la résistance fait explicitement référence à un passage du premier tome de l'Histoire de la sexualité et de quelques extraits d'entrevues accordées par Foucault. La leçon principale de Foucault à ce sujet est la suivante : la résistance ne tend pas à renverser le jeu de pouvoir qu'elle conteste, mais elle cherche plutôt à le modifier pour créer de nouvelles possibilités. Pour être en mesure de saisir la signification de cette leçon de Foucault, il est souhaitable de remettre en contexte la réflexion foucaldienne et de souligner un peu plus clairement l'importance de sa thèse.

L'analyse de Foucault dans le premier tome de l'Histoire de la sexualité prend place au sein d'une critique des différents mouvements de révolution ou de libération (féministe, homosexuel) des années 1970. La visée des différents mouvements révolutionnaires était de se libérer de l'oppression de l'hétérosexualité, du genre et d'une sexualité assujettie à des pratiques normatives. «La révolution est une méthode, est-il écrit dans le manifeste Gay Revolution Party Manifesto rédigé au début des années 70, par laquelle nous allons créer les conditions menant à la destruction du 
système de caste, de genre, et d'ainsi mener à la libération gaie de chaque personne. Ce sera un changement total qui atteindra les racines de l'ordre social actuel et qui détruira tous ses aspects qui répriment la liberté. » (Jay et Young 343) L'idée principale à la base des mouvements de libération homosexuelle et lesbienne ${ }^{5}$ est donc de détruire un système contraignant dont la présence met en péril la liberté véritable de chaque personne (homosexuelle et hétérosexuelle) et d'une sexualité naturelle. L'acte de libération ou de révolution permettra de mettre à nu une sexualité naturelle et d'ainsi dévoiler une existence humaine plus authentique.

La critique foucaldienne porte précisément sur deux éléments : tout d'abord, la possibilité de s'exclure totalement d'un système de normes, et ensuite l'existence d'une nature humaine se déployant au-delà de toutes normes. Les analyses de Foucault démontrent que nous ne pouvons jamais être «en position d'extériorité par rapport au pouvoir » (Foucault, Histoire 126) ou à un système de normes. Au lieu de vouloir se dégager complètement du jeu de pouvoir, ce que Foucault refuse catégoriquement, la seule chance que nous avons pour l'altérer est de pratiquer une résistance. Il n'y a donc pas, écrit Foucault, «par rapport au pouvoir un lieu du grand Refus - âme de la révolte, foyer de toutes les rébellions, loi pure du révolutionnaire. Mais des résistances qui sont des cas d'espèce [...]; par définition, elles ne peuvent exister que dans le champ stratégique des relations de pouvoir. » $(126)^{6}$ Pour Foucault, la résistance ne représente pas un état passager visant une révolution dans un champ libéré

5 Le manifeste des Radicalesbians The Woman-Identified Woman met en scène explicitement une libération du soi : «C'est la primauté des rapports que les femmes entretiennent entre elles, des femmes créant une nouvelle conscience de soi et des autres qui est au cœur de la libération. Ensemble nous devons trouver, renforcer et valider notre authentique soi. [...] Nous nous percevons comme fondamentale, nous trouvons notre centre à l'intérieur de nous. Nous nous trouvons en train de fuir notre sentiment d'aliénation, d'être coupées, d'être derrière une fenêtre barrée, d'être incapables de montrer ce que nous savons être à l'intérieur de nous. Nous sentons une authenticité, nous sentons enfin que ce que nous sommes coïncide avec notre soi. Avec ce véritable soi, avec cette conscience, nous débutons une révolution pour mettre fin à l'imposition de toute identification coercitive et accomplir l'autonomie de l'expression humaine. » (Jay et Young $176 s q$.) Le dévoilement d'un soi authentique que tous les êtres humains partagent mettrait fin à l'oppression et permettrait à chacun de vivre en toute liberté. Véritable acte de dénudation, le dévoilement rendu possible par la libération signifie un retour à un soi dont l'existence a consisté jusqu'ici à être recouvert par des normes ou des contraintes. Au-delà de la seule cause homosexuelle ou lesbienne, la révolution conduit à la révélation de la véritable nature humaine.

${ }^{6}$ Foucault affirme entrevue : «il n'y a pas de relations de pouvoir sans résistances; que celles-ci sont d'autant plus réelles et plus efficaces qu'elles se forment là même où s'exercent les relations de pouvoir; la résistance au pouvoir n'a pas à venir d'ailleurs pour être réelle, mais elle n'est pas piégée parce qu'elle est la compatriote du pouvoir. Elle existe d'autant plus qu'elle est là où est le pouvoir; elle est donc comme lui multiple et intégrable à des stratégies globales. » (Foucault, « Pouvoirs et stratégies » 425) 
du pouvoir, elle joue plutôt à chaque fois au cœur du pouvoir. Il affirme en entrevue :

Voyez-vous, s'il n'y avait pas de résistance, il n'y aurait pas de rapports de pouvoir. Parce que tout serait simplement une question d'obéissance. Dès l'instant où l'individu est en situation de ne pas faire ce qu'il veut, il doit utiliser des rapports de pouvoir. La résistance vient donc en premier, et elle reste supérieure à toutes les forces du processus; elle oblige, sous son effet, les rapports de pouvoir à changer. Je considère donc que le terme de «résistance » est le mot le plus important, le mot-clef de cette dynamique. (Foucault, « Sexe, pouvoir » 1559 sq.)

La résistance se définit ainsi comme refus stratégique ${ }^{7}$ dont l'action vise une modification des rapports de pouvoir qui se jouent partout dans notre société.

Comme le stipule Foucault, le premier geste de la résistance en est un négatif : il faut dire non. «Dire non », affirme Foucault, «constitue la forme minimale de résistance. [...] Il faut dire non et faire de ce non une forme de résistance décisive. » (1560) La résistance naît ainsi d'une négation (non); mais la négation permet également l'existence de nouveaux jeux de pouvoir. Nous retrouvons ainsi ce qu'affirme Halperin : la résistance n'est pas purement négative, elle est également positive et créatrice.

La leçon de Foucault aura donc été de démontrer que la résistance ne tend pas à renverser le pouvoir, mais qu'elle cherche à le modifier. Cette leçon, reprise par la résistance queer, se situe au cœur de l'analyse conceptuelle de la queeritude. La résistance ne représente pas ainsi un élément secondaire à la formation du concept de «queer », il s'agit plutôt d'un élément constitutif central à sa compréhension. Cette manière de représenter la forme rebelle ou contestataire du queer met en scène, comme nous le verrons, une temporalité qui se déploie en tant que négation positive.

\section{Temporalité hétéronormative}

Avant d'en venir à l'analyse spécifique de la résistance temporelle queer, nous devons nous interroger à savoir ce contre quoi la queeritude résiste. Malgré le caractère anodin de cette interrogation, celle-ci permettra de mettre en place un second concept essentiel à la signification du concept de queer: l'hétéronormativité. En plus d'interroger la

\footnotetext{
${ }^{7}$ «En fait, voyez-vous, je pense que la résistance est un élément de ce rapport stratégique en quoi consiste le pouvoir. La résistance prend toujours appui, en réalité, sur la situation qu'elle combat. » (Foucault, « Sexe, pouvoir » 1560)
} 
signification de ce concept, cette analyse nous servira de clé pour l'étude de la temporalité queer. Comme nous pourrons le constater, l'hétéronormativité se fonde sur une temporalité précise contre laquelle se déploie une temporalité propre à la queeritude.

Le concept d'hétéronormativité peut aisément porter à confusion et être mal interprété. La raison qui explique cette confusion est causée principalement par la conjonction qui est à la base du concept : l'hétérosexualité et la normativité. Intuitivement, nous pouvons comprendre la signification de ce concept comme suit : l'hétérosexualité en tant que norme. La résistance queer se formerait ainsi pour combattre l'hétérosexualité en tant que norme imposée à tous. Cette compréhension intuitive représente un piège qu'il nous faut absolument éviter. À l'image de l'«hétérosexualité obligatoire » (Rich) ou de la "pensée straight» (Wittig), l'hétéronormativité symbolise bien plus qu'une critique de la pratique sexuelle entre un homme et une femme. Rappelons-nous que l'«hétérosexualité obligatoire», définie en tant qu' "institution » (Rich 17), vise la critique de tous les faits sociaux à la source de cette contrainte, et que la «pensée straight» se veut «une interprétation totalisante à la fois de l'histoire, de la réalité sociale, de la culture et des sociétés, du langage et de tous les phénomènes subjectifs. » (Wittig 71) On le constate rapidement, la critique de Rich et de Wittig, même si elle se fonde sur l'hétérosexualité (straight), vise principalement les institutions qui s'accolent à un mode de vie qui repose sur cette pratique sexuelle. L'hétérosexualité ne représente pas ainsi la visée principale de la critique amorcée par Rich et Wittig. Il en va de même pour le concept d'hétéronormativité. Ce concept, apparu sous la plume de Warner dans l'introduction au recueil de 1993 Fear of a Queer Planet, inclut une réflexion et une critique globale de la société, "parce que la logique de l'ordre sexuel est si profondément intégrée actuellement dans un large éventail d'institutions sociales et dans des valeurs les plus traditionnelles du monde. » (xiii)

L'hétéronormativité, devenu un concept de base en théorie queer, nous aide à situer les principales caractéristiques d'un mode de vie dont la portée vise l'ensemble des institutions de notre société : «L'hétéronormativité [...] est présente dans presque chaque aspect des formes et des dispositions de la vie sociale : la nationalité, l'État et la loi; le commerce, la médecine et l'éducation; en plus des conventions et des affects liés à la narrativité, aux relations amoureuses et aux autres espaces protégés de la culture. » (Warner et Berlant 194) Le cadre normatif propre à l'hétéronormativité s'inscrit dans un espace beaucoup plus large qui admet une réflexion sur l'existence de nos principales institutions. Nous naissons tous dans un milieu social où se partage une série de normes qui s'incarnent en nous. L'hétéronormativité est le terme générique qui permet 
de décrire le jeu complexe des normes produites par les institutions et dont l'effet se fait sentir dans notre vie.

Pour que l'hétéronormativité puisse être qualifiée de norme, elle doit pouvoir se reproduire, c'est-à-dire se transmettre de génération en génération et se baser sur la répétition et la reproduction de certains gestes, de certaines croyances et d'une certaine imagination. Le caractère contraignant d'une norme provient principalement du fait qu'elle se répète dans le temps jusqu'à devenir un précepte quasi naturel. La temporalité propre à l'hétéronormativité se présente ainsi en tant que reproduction de génération en génération d'un même mode de vie dont la principale institution se dévoile dans la famille hétérosexuelle. Halperin énumère et décrit les principales normes au fondement de la famille hétérosexuelle :

Selon ces normes, la dignité et la valeur de la vie humaine trouvent leur expression dans une forme particulière d'intimité : le couple. Une telle existence, afin d'être, nécessite une vie domestique stable, partagée avec une autre personne sensiblement du même âge, mais de genre et de sexe différent [...] dans un amour exclusif, dyadique, non-commercial qui est réalisé dans l'espace conjoint de la maison, établi et consolidé par le droit de propriété et d'autres formes de richesses qui peuvent être transmises de génération en génération. Intimité, amour, amitié, solidarité, sexualité, reproduction, éducation des enfants, succession de générations, gardiennage, soutien mutuel, espace de vie commun, partage des finances, droit de propriété et de la vie privée vont ensemble et ne devraient pas être morcelés entre différentes relations. Ils se combinent pour constituer une forme sociale unique, de valeur incomparable, plus ou moins obligatoire. (Halperin, How to be Gay 450)

Cette série de normes, qui définit en grande partie la sphère de l'hétéronormativité, acquiert valeur de précepte uniquement en raison de sa reproduction générationnelle et du même coup à partir d'une temporalité qui se fonde sur la continuité entre le passé, le présent et le futur. L'hétéronormativité donne une orientation à notre existence dont l'une des finalités est de perpétuer les normes familiales du passé dans le futur. L'orientation temporelle propre à l'hétéronormativité donne ainsi une signification ${ }^{8}$, une direction à notre existence en nous imposant un

\footnotetext{
${ }^{8}$ Warner se sert du concept de repro-narrativité pour décrire l'un des aspects importants de la temporalité hétéronormative : "l'idée que nos vies possèdent en quelque sorte plus de sens en étant incorporées dans une narration de succession générationnelle. » (Warner, «Introduction » 7)
} 
mode de vie dans lequel les normes familiales forment le cadre général de la vie sociétale?

\section{Chrononormativité et hétéronormativité}

On aura compris qu'en décrivant la temporalité hétéronormative en tant que reproduction d'un mode de vie, nous ne faisons pas référence à la temporalité que nous lisons sur les horloges ou que nous pouvons mesurer historiquement. Comprise en tant que mode de vie, la temporalité hétéronormative s'inscrit à même le corps, l'imagination et l'horizon d'attente de l'individu. Cette temporalité est donc ce qui forme l'existence de celui ou de celle qui l'habite et ce qui donne une signification à cette existence. Elizabeth Freeman, dans son ouvrage Time Binds, emploie le terme de chrononormativité pour décrire la temporalité qui, en plus de devenir une norme ou une habitude quotidienne, se grave dans le corps :

La chrononormativité est un mode d'implantation, une technique à partir de laquelle des pouvoirs institutionnels en viennent à ressembler à des faits somatiques. Les horaires, les calendriers, les fuseaux horaires et même les montres inculquent ce que le sociologue Eviatar Zerubavel nomme «les rythmes cachés », les formes de l'expérience temporelle qui semblent naturelles [...]. (Freeman 3)

La chrononormativité se résume à l'incorporation de schèmes temporels à la vie la plus intime.

Freeman se tourne vers l'expérience temporelle du travail pour décrire l'effet corporel ou affectif de la temporalité. "L'avènement du travail salarié, par exemple, entraîne une violente retemporalisation des corps, corps qui étaient accordés aux rythmes saisonniers du travail de la terre. » (3) Par cet exemple, Freeman démontre que le corps s'habitue, par le travail, à une certaine temporalité : travailler du lundi au vendredi de $8 \mathrm{~h}$ à $17 \mathrm{~h}$ ou travailler au rythme des saisons représentent deux expériences temporelles différentes et deux expériences corporelles différentes. Cette temporalité s'incorpore, elle devient l'expérience même du corps.

Nous pouvons rapprocher cette description de la temporalisation des corps avec ce que Pierre Bourdieu décrit comme habitus. L'habitus est ce qu'un individu acquiert par l'incorporation et la répétition de certains

\footnotetext{
${ }^{9}$ Halperin résume bien le caractère temporel et l'orientation propre à l'hétéronormativité : «L'hétéronormativité représente les privilèges d'un horizon d'attente normatif pour une vie humaine florissante. Elle génère une éthique de reproduction personnelle et collective, et implique une orientation pour le futur. Elle produit une esthétique pour l'être social, esthétique qui se fixe à la forme d'une vie et lui donne une beauté et une valeur. Elle incarne une structure imaginative qui confère une signification à la forme de l'existence individuelle. » (How to Be Gay 451)
} 
faits et gestes. Bourdieu a une expression pour décrire cette incorporation et la répétition par l'habitus : il dit que nous «apprenons par corps », c'est-à-dire que c'est le corps même qui incorpore une manière de vivre, qui devient lui-même ce qu'il incorpore : "Ce qui est appris par corps ", écrit Bourdieu, «n'est pas quelque chose que l'on a, comme un savoir que l'on peut tenir devant soi, mais quelque chose que l'on est. » (Bourdieu 123) Ainsi, l'expérience temporelle qu'incorpore l'individu par la répétition de certains faits et gestes représente ce qu'il est, il représente une part essentielle de la vie la plus intime de l'individu ${ }^{10}$.

L'analyse de la chrononormativité permet, dans un premier temps, de rendre compte du caractère construit ou acquis de notre expérience temporelle et, dans un second temps, de montrer de quelle manière nous sommes en mesure d'acquérir une telle expérience. La chrononormativité explique la présence, en tant que mode de vie, de la temporalité hétéronormative au cœur de l'existence contemporaine. En mettant en valeur le caractère temporel des principaux éléments qui fondent l'hétéronormativité, Halberstam réussit à nous faire comprendre le caractère proprement chrononormatif de la famille, de la reproduction et de l'héritage : "Le temps de la famille désigne l'horaire normatif de la vie quotidienne (se coucher tôt, se lever tôt) qui accompagne la pratique de l'éducation des enfants. Cet horaire, régi par un ensemble de besoins imaginaires des enfants, concerne les croyances sur la santé des enfants et de l'environnement sain pour l'éducation de ceux-ci. » (Halberstam 5) L'horaire familial devient la norme temporelle à partir de laquelle nous pouvons juger du bien-fondé de tout autre horaire possible. Le temps employé pour l'éducation des enfants est un temps porté vers l'avenir, un temps futur dans lequel l'enfant d'aujourd'hui devra à son tour éduquer ses propres enfants, et ainsi de suite.

La chrononormativité propre à l'hétéronormativité se déploie dans la reproduction d'une temporalité qui se fonde sur l'éducation des enfants et sur la transmission de certaines valeurs propres à la vie familiale.

\footnotetext{
${ }^{10}$ Nous pourrions ajouter à cette analyse de la chrononormativité et de l'habitus le concept de performativité tel que décrit par Butler. Sans entrer ici dans les détails de la conception butlérienne de la performativité, nous pouvons tout de même souligner que pour qu'une norme s'incorpore à un corps, elle doit se répéter à partir de gestes du corps : « la performativité n'est pas un acte unique, mais une répétition et un rituel, qui produit ses effets à travers un processus de naturalisation qui prend corps, un processus qu'il faut comprendre, en partie, comme une temporalité qui se tient dans et par la culture. » (Butler, Trouble 36) Il est intéressant de noter qu'à la suite de ce passage, Butler établit, en note de bas de page, le rapport entre cette définition de la performativité et celle de l'habitus qu'on retrouve chez Bourdieu. Elle écrit : «L'idée de dimension rituelle de la performativité rejoint en partie la notion d'habitus dans le travail de Pierre Bourdieu, ce que je n'ai réalisé qu'après avoir écrit ce livre. J'ai essayé par la suite d'expliquer cette affinité dans le dernier chapitre de Excitable Speech. A Politics of the Performative. » (36) Cf. Butler, Excitable Speech 127-163 et «Performativity's Social Magic ».
} 
Edelman nomme la temporalité propre à l'hétéronormativité par l'expression de «futurisme reproductif» et insiste pour souligner le caractère normatif de la temporalité familiale en décrivant celle-ci comme le seul véritable principe communautaire admis en société. Le « futurisme reproductif» porte vers le seul avenir possible : «Cet Enfant reste le perpétuel horizon de toute politique reconnue, le bénéficiaire fantasmatique de toute intervention politique. » (Edelman 287) Le «futurisme reproductif » a comme visée principale la transmission d'un héritage dont la portée transgresse les seules limites de la famille pour rendre possibles également les fondements de notre vie politique. Halberstam écrit à ce sujet :

Le temps de l'héritage se rapporte à une vue d'ensemble du temps générationnel dans lequel des valeurs, la richesse, les biens et la morale sont transmis à travers les liens familiaux d'une génération à l'autre. Cette situation permet également de rattacher la famille au passé historique de la nation, et de rattacher la famille à la stabilité future tant de celle-ci que de la nation. (Halberstam 5)

Ce que cette transmission permet de mettre au jour est la continuité qui est au cœur de la temporalité hétéronormative. Cette temporalité se comprend en tant que chaîne : chaque génération se rapporte à celle qui l'a précédée et à celle qui suivra. Il y a ainsi une continuité, et cette continuité, cette séquence, suit une ligne temporelle : du passé au présent jusqu'au futur. Ahmed donne un éclairant aperçu de cette temporalité lorsqu'elle décrit l'expérience de la vie familiale en tant que ligne à suivre :

Le couple hétérosexuel devient un «point» le long d'une ligne, ligne qui est transmise à l'enfant comme héritage ou comme toile de fond [Background]. La toile de fond n'est pas simplement derrière l'enfant : elle est ce que l'enfant est invité à aspirer. La toile de fond, définie de cette façon, peut nous orienter vers le futur : elle est ce que l'enfant est invité à désirer en acceptant la ligne familiale comme son propre héritage. Il y a une pression pour hériter de cette ligne, une pression qui peut parler le langage de l'amour, du bonheur et du soin [care], qui nous pousse le long de chemins précis. (Ahmed, Queer Phenomenology 90)

En reproduisant le présent dans le futur, l'enfant aspire à réaliser un désir. Ce désir est ce qui l'oriente vers le futur, futur déjà tracé et sur le point d'être accompli. Que se passe-t-il, toutefois, lorsque l'enfant (ou la personne adulte) ne désire pas reproduire l'héritage légué par la cellule familiale et, plus généralement, par le mode de vie hétéronormatif? Que signifie dire «non » à ce qui nous est transmis dès notre plus jeune âge? Avec ce «non » s'ouvre une autre expérience du futur qui se fonde sur un 
désir qui n'aspire pas à perpétuer un futur déjà écrit et qui ne réserve quasi aucune surprise.

\section{Futurité queer : désir et utopie}

Dire non à l'héritage, c'est résister à une temporalité qui se fonde sur la reproduction. Résister ne consiste pas uniquement dans le fait de dire non; cela donne accès à un futur encore indéterminé, potentiellement nouveau, garant de nouvelles possibilités. La résistance queer a pour conséquence d'ouvrir un champ de possibilités absent du système hétéronormatif et dont la présence permet d'espérer un nouveau futur. Par le biais de la résistance, on a ainsi accès à un futur indéfini que nous pouvons qualifier de temporalité queer : «Les temporalités queer, visibles dans la forme de l'interruption [...] sont des points de résistance à cet ordre temporel [hétéronormatif] [...] des points de résistance qui proposent d'autres possibilités de vivre en relation avec un passé, un présent et un futur » (Freeman xxii). Ces points de résistance temporelle, loin d'être une rébellion abstraite, prennent plutôt place au cœur de la vie affective par l'entremise d'un désir. Nous pouvons affirmer que le désir est la source de la résistance queer et une critique de la temporalité hétéronormative. Il ne faut pas se surprendre outre mesure de l'importance que nous accordons au désir dans notre analyse de la temporalité queer et ce, pour la simple raison que cette dernière s'incarne dans la vie affective et dans l'action de ceux et celles pratiquant la queeritude.

Le lien entre la temporalité queer et le désir est explicitement établi par José Esteban Muñoz dans Cruising Utopia. The Then and There of Queer Futurity. La futurité queer exposée par Muñoz se fonde sur une négativité, c'est-à-dire sur une critique radicale du «ici et maintenant» dans lequel nous vivons. Pour Muñoz, si la personne queer critique le «ici et maintenant », c'est parce qu'elle croit qu'un changement est possible et que de nouvelles possibilités peuvent naître dans le futur. «Être queer est cette chose qui nous fait sentir que ce monde ne suffit pas, qu'il y manque effectivement quelque chose. » (Muñoz 1) Cette expérience du manque acquiert une réalité seulement à partir du désir que vit intensément la personne queer. «La queeritude [...] se fonde sur une économie du désir et du désirable. Ce désir est à tout moment dirigé sur cette chose qui n'est pas encore là, sur des objets et des moments qui brûlent de promesse et d'anticipation. » (26) C'est par le désir de vivre autrement, ou même de pouvoir vivre autrement, qu'une visée futuriste est rendue possible : «La queeritude [queerness] devrait et pourrait porter un désir pour une autre manière d'être dans le monde et dans le temps » (Muñoz 96). Muñoz perçoit dans ce désir une potentialité, une force que nous devons comprendre de deux manières: tout d'abord, en tant que ce qui nous 
pousse à vouloir changer le «ici et maintenant», et également en tant qu'ouverture à de nouvelles possibilités.

En plus d'être un affect (désir), la temporalité queer possède un caractère performatif : être queer, écrit Muñoz, «est également performatif parce que ce n'est pas simplement une manière d'être mais également un faire pour et vers le futur. » (1) Le désir incarné de changer le « ici et maintenant » incite à l'action, c'est-à-dire à combler le manque qui est au fondement de l'insatisfaction de la situation actuelle.

Pour pouvoir seulement imaginer qu'il existe d'autres manières de vivre, nous devons être en mesure de nous inventer un lieu et un temps qui n'existe pas encore. Si Muñoz entreprend une étude de l'utopie, c'est parce que l'utopie est ce qui lui permet d'interroger le présent : «L'utopie est un mode critique idéaliste qui nous rappelle que quelque chose manque, que le présent et la présence ne sont pas assez. » (100) Ainsi, si Muñoz se tourne vers une étude de l'utopie pour traiter d'un pan essentiel de la temporalité queer (le futur), c'est parce qu'elle lui permet de critiquer le «ici et maintenant» que représente l'hétéronormativité : «L'utopie nous laisse imaginer un espace en-dehors de l'hétéronormativité. Cela nous permet de conceptualiser de nouveaux mondes et de nouvelles réalités qui ne sont pas irrévocablement liés à l'épidémie du VIH/SIDA et à l'homophobie institutionnalisée de l'État. » (35) Pour pouvoir imaginer, conceptualiser et mettre en action de nouvelles réalités et de nouvelles relations humaines, il faut tenter de transgresser les limites de cet «ici et maintenant» hétéronormatif. Il faut être en mesure d'imaginer autre chose qui n'est pas encore ici et maintenant, il faut imaginer un lieu et un temps encore inaccessibles, c'est-à-dire une utopie.

Le futur doit se comprendre en tant que potentialité, c'est-à-dire comme ouverture à ce qui n'est pas ici et maintenant. L'espérance ressentie à la base de l'utopie queer doit s'interpréter à partir de cette possibilité potentielle du futur. Il faut que le futur s'ouvre, permette d'espérer une autre réalité : «La futurité queer que je décris n'est pas une fin, mais une ouverture ou un horizon. » (91) La futurité queer ne doit pas se comprendre en tant que programme, mais bien plutôt en tant qu'ouverture d'un horizon dans lequel tout est possible. Décrite de cette manière, la futurité queer ne cessera jamais d'être indéterminée et ouverte à l'inédit.

La futurité queer s'oppose fondamentalement à la répétition et à la reproduction. L'ouverture vers l'inédit proposée par le jeu de la queeritude ne tend pas à se transformer en un état fixe ou en une identité définie et circonscrite. L'indétermination de la futurité queer représente plutôt un état inconfortable dans lequel se crée de l'inédit. L'acte de résistance, que nous avons décrit plus tôt comme étant le cœur du concept de queeritude, 
s'applique parfaitement au travail de la futurité : elle se présente comme négation du «ici et maintenant» tout en permettant la venue de ce qui n'est pas encore présent. Le déploiement de la futurité s'impose ainsi comme une pratique transitoire dont le moteur se trouve dans le désir de vivre autrement que dans le quadrillage serré de l'hétéronormativité.

***

L'intérêt que nous avons porté ici à la queeritude vise une analyse conceptuelle et non pas une description historico-sociologique. Nous ne sommes pas sans savoir qu'il existe des personnes qui se définissent en tant que queer et que des communautés se forment sous cette appellation. Nous reconnaissons également que le terme «queer» reçoit depuis quelques années une attention particulière et qu'il est devenu commun de le percevoir comme synonyme de gai, lesbienne, trans et bi. Le problème qui surgit lorsque l'on transforme la queeritude en identité ou en pratique sexuelle normée est que l'on perd la vivacité et la force subversive que l'on retrouve dans les actions politiques d'ACT UP ou de Queer Nation et dans les principaux textes du corpus des études queer. La crainte que la queeritude s'intègre à un système de normes et qu'elle devienne une identité comme une autre est devenue réalité et elle sape l'élan perturbateur du queer.

Il peut paraître paradoxal de procéder à une analyse conceptuelle d'un terme dont on ne peut pas circonscrire totalement la nature. Notre insistance à décrire la queeritude comme concept dynamique et transitoire nous pousse à admettre que le principal nous échappe, que le mouvement, qui est le cœur de la queeritude, ne peut être saisi. On doit respecter cette difficulté majeure, mais le travail d'analyse conceptuelle montre tout de même les limites à partir desquelles on peut comprendre la spécificité du queer. La queeritude comme concept dynamique et transitoire a tous les aspects d'un concept proprement temporel. Étant toujours en mouvement (dynamique) et étant à la limite de ce qui est et de ce qui n'est pas (transitoire), la queeritude s'inscrit toujours dans une temporalité qui la porte vers l'avant. La futurité queer ne représente pas uniquement un moment de la queeritude, elle en est le cour et le sang. La queeritude se présente comme ouverture vers l'inconnu, l'inédit.

La futurité ne procède pas sans l'apport essentiel de l'action ou de la performance de la résistance. Décrit comme négativité positive, la résistance doit également se comprendre comme concept dynamique et transitoire, car lui aussi se porte vers l'avant, vers de nouvelles possibilités. La potentialité propre à la résistance en fait un concept temporel tourné vers une futurité encore inexplorée. Le lien que nous établissons entre la résistance, la futurité et le caractère dynamicotransitoire de la queeritude donne accès à une définition conceptuelle du 
terme « queer ». Au cœur de cette conceptualité se dévoile une temporalité dont l'importance ne peut être passée sous silence. L'indétermination propre à la queeritude s'explique par la présence de cette temporalité qui incite celle-ci à ne jamais rester en place.

\section{Textes cités}

AHMED, Sarah, Queer Phenomenology. Orientations, Objects, Others, Durham, Duke University Press, 2006.

—, The Promise of Happiness, Durham, Duke University Press, 2010.

BERGER, Anne-Emmanuelle, Le grand théâtre du genre. Identités, sexualités et féminisme en " Amérique », Paris, Belin, 2013.

BOURCIER, Marie-Hélène, Queer Zones. Politique des identités sexuelles et des savoirs, Paris, Amsterdam, 2006.

BOURDIEU, Pierre, Le sens pratique, Paris, Minuit, 1980.

BUTLER, Judith, Ces corps qui comptent. De la matérialité et des limites discursives du sexe, trad. C. Nordmann, Paris, Amsterdam, 2009.

-, Excitable Speech. A Politics of the Performance, New York, Routledge, 1997.

—, «Performativity's Social Magic », in R. Shusterman (dir.), Bourdieu. A Critical Reader, Oxford, Blackwell, 1999, p. 113-128.

-, Trouble dans le genre. Le féminisme et la subversion de l'identité, trad. É. Fassin, Paris, La Découverte, 2005.

CVETKOVICH, Ann, An Archive of Feelings. Trauma, Sexuality, and Lesbian Public Cultures, Durham, Duke University Press, 2003.

DINSHAW, Carolyn, Getting Medieval. Sexualities and Communities, Pre- and Postmodern, Durham, Duke University Press, 1999.

- How Soon is Now? Medieval Texts, Amateur Readers, and the Queerness of Time, Durham, Duke University Press, 2012.

DUGGAN, Lisa, The Twilight of Equality? Neoliberalism, Cultural Politics, and the Attack on Democracy, Boston, Beacon Press, 2003. 
EDELMAN, Lee, L'impossible homosexuel, trad. G. Le Gaufrey, Paris, Epel, 2013.

ENG, David L., The Feeling of Kinship. Queer Liberalism and the Racialization of Intimacy, Durham, Duke University Press, 2010.

FERGUSON, Roderick A., Aberrations in Black. Toward a Queer of Color Critique, Minneapolis, University of Minnesota Press, 2004.

FOUCAULT, Michel, Histoire de la sexualité, vol. 1: La volonté de savoir, Paris, Gallimard, 1976.

—, «Pouvoirs et stratégies », in M. Foucault, Dits et écrits, vol. 2, Paris, Gallimard, 2001, p. 418-428.

—, «Sexe, pouvoir et politique de l'identité », M. Foucault, Dits et écrits, vol. 2, Paris, Gallimard, 2001, p. 1554-1565.

FRECCERO, Carla, Queer/Early/Modern, Durham, Duke University Press, 2006.

FREEMAN, Elizabeth, Time Binds. Queer Temporalities, Queer Histories, Durham, Duke University Press, 2010.

GOULD, Deborah B., Moving Politics. Emotion and ACT UP's Fight Against AIDS, Chicago, University of Chicago Press, 2009.

HALBERSTAM, Judith, In a Queer Time and Place. Transgender Bodies, Subcultural Lives, New York, New York University Press, 2005.

HALLEY, Janet et Andrew PARKER, «Introduction », in J. Halley et A. Parker (dir.), After Sex? On Writing since Queer Theory, Durham, Duke University Press, 2011, p. 1-14.

HALPERIN, David M., Saint Foucault, trad. D. Éribon, Paris, Epel, 2000.

—, How to be Gay, Cambridge (MA), Harvard University Press, 2012.

JAY, Karla et Allen Young, Out of the Closet. Voices of Gay Liberation, New York, New York University Press, 1992.

LOVE, Heather, Feeling Backward. Loss and the Politics of Queer History, Cambridge (MA), Harvard University Press, 2007.

MCCALLUM, E.L. et Mikko TUHKANEN (dir.), Queer Times, Queer Becoming, Albany, State University of New York Press, 2011. 
MUÑOZ, José Esteban, Cruising Utopia. The Then and There of Queer Futurity, New York, New York University Press, 2009.

NEALON, Christopher, Foundlings. Lesbian and Gay Historical Emotion Before Stonewall, Durham, Duke University Press, 2001.

PUAR, Jasbir K., Terrorist Assemblages. Homonationalism in Queer Times, Durham, Duke University Press, 2007.

REDDY, Chandon, Freedom with Violence. Race, Sexuality, and the US State, Durham, Duke University Press, 2011.

$\mathrm{RICH}$, Adrienne, «La contrainte à l'hétérosexualité et l'existence lesbienne », trad. C. Delphy et E. de Lesseps, Nouvelles questions féministes, $\mathrm{n}^{\circ} 1,1981$, p. 15-43.

SEDGWICK, Eve Kosofsky, Tendencies, Durham, Duke University Press, 1993.

-, Touching Feeling. Affect, Pedagogy, Performativity, Durham, Duke University Press, 2003.

—, Épistémologie du placard, trad. M. Cervulle, Paris, Amsterdam, 2008.

WARNER, Michael, «Introduction: Fear of a Queer Planet », Social Text, $n^{\circ} 29,1991$, p. 3-17.

—, Publics and Counterpublics, New York, Zone Books, 2005.

—, «Queer and Then », The Chronicle of Higher Education, janv. 2012, n.p.

WITTIG, Monique, La pensée straight, Paris, Balland, 2001, p. 65-76. 